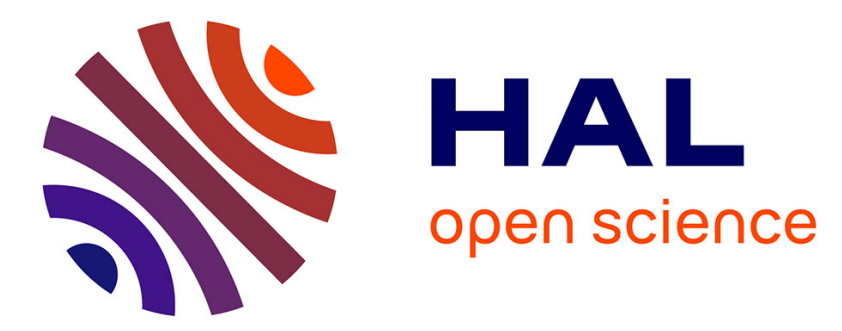

\title{
Orientational ordering in a dilute system of classical interacting quadrupoles: (N2-Ar) solid mixtures
}

\author{
Daniel Estève, N.S. Sullivan, M. Devoret
}

\section{To cite this version:}

Daniel Estève, N.S. Sullivan, M. Devoret. Orientational ordering in a dilute system of classical interacting quadrupoles: (N2-Ar) solid mixtures. Journal de Physique Lettres, 1982, 43 (22), pp.793-799. 10.1051/jphyslet:019820043022079300 . jpa-00232127

\section{HAL Id: jpa-00232127 https://hal.science/jpa-00232127}

Submitted on 1 Jan 1982

HAL is a multi-disciplinary open access archive for the deposit and dissemination of scientific research documents, whether they are published or not. The documents may come from teaching and research institutions in France or abroad, or from public or private research centers.
L'archive ouverte pluridisciplinaire HAL, est destinée au dépôt et à la diffusion de documents scientifiques de niveau recherche, publiés ou non, émanant des établissements d'enseignement et de recherche français ou étrangers, des laboratoires publics ou privés. 
Classification

Physics Abstracts

$31.70 \mathrm{~K}-64.70-76.60$

\title{
Orientational ordering in a dilute system of classical interacting quadrupoles : $\left(\mathbf{N}_{2}-\mathbf{A r}\right)$ solid mixtures
}

\author{
D. Estève, N. S. Sullivan and M. Devoret \\ DPh-G/PSRM, CEN-Saclay, 91191 Gif-sur-Yvette Cedex, France
}

(Reçu le 11 août 1982, accepté le 24 septembre 1982)

\begin{abstract}
Résumé. - Nous présentons une étude RMN d'un mélange solide $\left(\mathrm{N}_{2}-\mathrm{Ar}\right)$ pour $X_{\mathrm{N}_{2}}=0,67$. Dans ce système, les molécules d'azote peuvent être considérées comme des rotateurs classiques répartis au hasard sur un réseau hexagonal compact et couplés par des interactions anisotropes, de courte portée, du type quadrupôle-quadrupôle.

L'étude RMN de l'ordre onentationnel dans ce système, quı combıne de façon originale la frustration et le désordre, montre qu'à basse température les molécules d'azote adoptent des orientations aléatoires essentiellement fixes dans le temps, tout mouvement de réorientation s'effectuant à des fréquences inférieures à $10^{-2} \mathrm{~Hz}$. Ce régime, dit de verre orientationnel, s'installe progressivement lorsque la température diminue sans qu'une transition soit détectée. On pense cependant qu'il s'agit là d'un phénomène très coopératif.
\end{abstract}

\begin{abstract}
We present an NMR study of a $\left(\mathrm{N}_{2}-\mathrm{Ar}\right)$ solid mixture $\left(X_{\mathrm{N}_{2}}=0.67\right)$ in which the $\mathrm{N}_{2}$ molecules can be considered as almost classical rotators randomly distributed on an h.c.p. lattice. The orientational ordering of the molecules due to their short range highly anisotropic quadrupolequadrupole interactions presents an original case study of the combined effects of frustration and disorder.

At low temperatures the NMR results show that the $\mathrm{N}_{2}$ molecules adopt random frozen-in orientations for which any residual motion occurs at frequencies less than $10^{-2} \mathrm{~Hz}$. This regime, called an onentational glass, grows continuously on cooling without any detectable transition but it is nevertheless believed to result from a highly cooperative phenomenon.
\end{abstract}

1. Introduction - While ordinary glasses represent one of the oldest materials known to man, a comprehensive understanding of the formation of glasses has not yet been achieved. For this reason, interest has become focussed on conceptually simpler systems which are in many ways closely related to the general problem of glasses. The study of the so-called spin-glasses [1] has brought out the key role of the interplay between frustration and disorder in these compounds. Dilute systems of interacting quadrupoles, such as ortho-para $\mathrm{H}_{2}$, para-ortho $\mathrm{D}_{2}$, and $\mathrm{N}_{2}-\mathrm{Ar}$ solid mixtures, which incorporate in a simple way both frustration and disorder, have therefore received a continuously growing interest. Frustration in these systems arises from the topological impossibility of ensuring the minimum possible energy for all pairs of neighbouring quadrupoles. This is so for any configuration of quadrupoles on any $3 \mathrm{D}$ lattice. In spite of this conflict, pure systems undergo orientational phase transitions to long range ordered phases which have rather complicated configurations but nevertheless achieve the best energy compromise. For example, on the f.c.c. lattice, one observes a four sublattice $\mathrm{Pa} 3$ structure $[2,3]$.

When some of the quadrupole bearing molecules are randomly replaced by spherical molecules or atoms, which just play the role of an inactive diluant from the orientational point of view, 
the best energy compromise is not achieved for a long range ordered structure and the above transition no longer occurs. The study of solid ortho-para hydrogen [3-11] and deuterium [12] mixtures (in which the quadrupole bearing molecules, ortho $\mathrm{H}_{2}$ and para $\mathrm{D}_{2}$ are $J=1$ quantum rotators) has shown that for quadrupole concentrations such that the lattice remains h.c.p. at all temperatures [13] $(X \lesssim 55 \%)$ a new regime referred to as a quadrupolar glass [4] is encountered at low temperatures.

This regime is characterized by (i) a broad non-uniform distribution of orientational order parameters (which measure to what extent the molecular orientations are localized); this distribution evolves smoothly with the temperature, (ii) a substantial slowing-down of the molecular reorientations $[8,10]$ on lowering the temperature, and (iii) a heat capacity varying linearly with the temperature [11]. An important unknown for these systems is however the importance of orientational quantum correlations between molecules. In order to understand the relevance of these quantum effects for the realization of a quadrupolar glass regime, we have studied $\mathrm{N}_{2}-\mathrm{Ar}$ mixtures, in which the $\mathrm{N}_{2}$ molecules can be considered as almost "classical " quadrupoles. We report in this letter an NMR investigation of the orientational ordering in this system for $X_{\mathrm{N}_{2}}=0.675$, that is in the concentration regime for which previous $X$-ray studies [14] have shown that (i) the lattice remains hexagonal at all temperatures and (ii) there is no phase transition towards a long range orientationally ordered phase, as confirmed by specific heat studies [15].

2. NMR experiments - We have studied the NMR of a sample containing ${ }^{15} \mathrm{~N}$ nuclei [16] $(I=1 / 2)$ instead of the NQR of the common isotope ${ }^{14} \mathrm{~N}(I=1)$ since the large quadrupolar coupling constant of the ${ }^{14} \mathrm{~N}_{2}$ molecule would give rise to very large linewidths as soon as disorder is introduced in the system. Two molecular species of ${ }^{15} \mathrm{~N}_{2}$ exist, ortho molecules $(I=1)$ and para molecules $(I=0)$. Since the difference in symmetry of the orbital functions plays no role at the temperatures being considered here, $75 \%$ of the nitrogen molecules are ortho molecules whose nuclear magnetic resonance can be observed. It provides a powerful tool for studying their orientational state since the secular part of the intramolecular nuclear Hamiltonian $H_{\mathrm{n}}$ depends on the angle $\theta_{t}$ between the molecular axis $\hat{R}_{t}$ and the magnetic field applied along the $\hat{z}$ axis :

$$
H_{\mathrm{n}_{i}}=P_{2}\left(\theta_{i}\right)\left[-D\left(I_{t z}^{2}-\frac{2}{3}\right)+\chi I_{l z}\right]
$$

where : $P_{2}\left(\theta_{1}\right)=\frac{1}{2}\left(3 \cos ^{2} \theta_{1}-1\right), D / 2 \pi=1.38 \mathrm{kHz}$ is the intramolecular dipolar interaction and $\chi=\left(4 \times 10^{-4}\right) \omega_{0}$ is the asymmetric chemical shift proportional to the Larmor frequency $\omega_{0}$. In order to analyse the spectra, we shall assume that each molecule $i$ undergoes a rapid rotational motion (compared to the relevant NMR frequencies $D$ and $\chi$ ) about a fixed symmetry axis $\hat{\zeta}_{1}$ (at angle $\alpha_{2}$ with the field). In this case, the NMR spectrum is determined by the statistical average of the $P_{2}\left(\theta_{t}\right)$ which obeys :

$$
\left\langle P_{2}\left(\theta_{1}\right)\right\rangle=P_{2}\left(\alpha_{\imath}\right) \cdot x_{\imath}
$$

where the quadrupolarization $x_{i}$ measures to what extent the molecule $(i)$ is aligned along $\hat{\xi}_{i}$ :

$$
\boldsymbol{x}_{1}=\left\langle\boldsymbol{P}_{2}\left(\gamma_{2}\right)\right\rangle \text { with } \gamma_{t}=\left(\hat{\boldsymbol{R}}_{i}, \hat{\zeta}_{t}\right) .
$$

The NMR spectrum of the molecule $(i)$ consists of two lines shifted from the Larmor frequency by :

$$
\Delta \omega_{\imath}=P_{2}\left(\alpha_{\imath}\right) \cdot x_{1}( \pm D+\chi) .
$$

For a powder sample (uniform distribution of the $\alpha_{1}^{\prime}$ 's), each value of $x$ gives rise to an asymmetric Pake doublet (shown in the inset of figure 1) of width proportional to $x$. We have inferred 


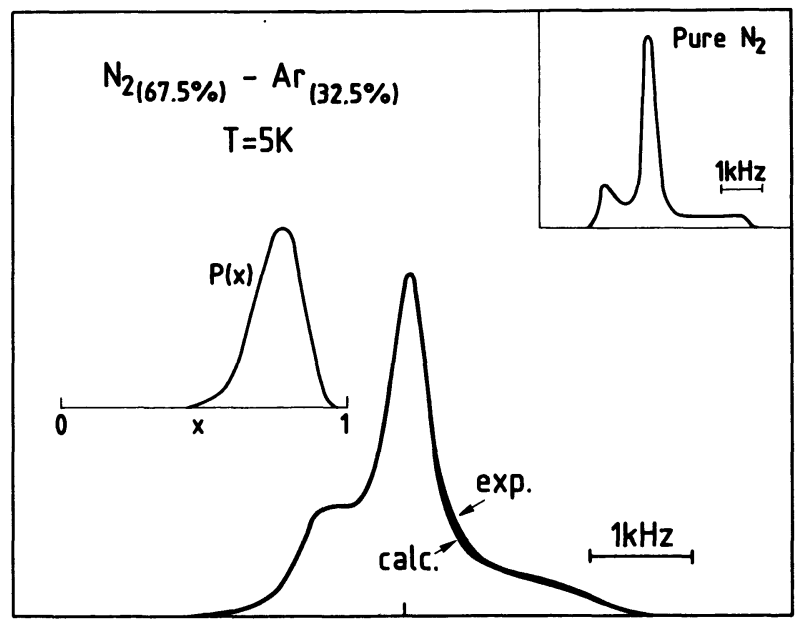

Fig. 1. - Comparison between the experimental NMR line shape recorded at a frequency of $2.9 \mathrm{MHz}$ for a $\left({ }^{15} \mathrm{~N}_{2} 67.5 \%-\mathrm{Ar}_{32.5 \%}\right)$ sample at $5 \mathrm{~K}$ and the calculated one using the quadrupolarization distribution function $P(x)$ displayed. The line shape of pure ${ }^{15} \mathrm{~N}_{2}$ (cubic phase, $P a 3$ structure) recorded under the same conditions is shown in the inset. It corresponds to the distribution $P(x)=\delta(x-0.866)$.

the distribution function $P(x)$ for the $x_{i}$ 's from the NMR line shapes following recently developed procedures $[5,7]$ which have provided a satisfactory fit to the data as shown in figure 1 . (Let us note here that the consideration of non-axial quadrupolarizations would not change our conclusions.)

The variation of the distribution $P(x)$ with the temperature is shown in figure 2 . The most remarkable conclusion is that there is a continuous growth of $P(x)$ towards large quadrupolarizations as the temperature is reduced without any detectable discontinuity : the molecular orientations become progressively more and more localized on cooling from $20 \mathrm{~K}$ to $10 \mathrm{~K}$, which corresponds to the temperature range for which an excess in the specific heat has been observed [15]. No further evolution is detected below $7 \mathrm{~K}:$ at these temperatures, the departure of the quadrupolarizations from the value $x=1$ is a residual quantum effect. This evolution is qualitatively similar to the behaviour observed for ortho-para hydrogen mixtures $[5,7]$ and appears to be a general feature of dilute systems of interacting quadrupoles regardless of their quantum character.

We now turn to the evaluation of the time scale over which the picture of orientational states with fixed local axes $\hat{\zeta}_{i}$ can be considered as correct. We know that this time scale is greater than $10 \mathrm{~ms}$ since the line shapes are not in the intermediate motional narrowing regime. This limit is however too short to assess whether the molecular orientational states are well defined in time. In order to detect any residual motion, we have used pulse NMR methods specially conceived for that purpose and published elsewhere $[17,18]$. The first method that we shall only briefly discuss consists of measuring the damping factor of the solid echo following two RF pulses separated by a time $\tau$ : since the damping of the solid echoes due to the dipolar intermolecular interactions is known precisely, an appreciable overdamping due to a possible slow motion of the axes $\hat{\zeta}_{i}$ (or to a possible slow time dependence of the quadrupolarizations $x$ ) can be detected if this evolution takes place in a time less than $\left(D^{2} \tau^{3}\right)$.

Our investigations of solid echoes in this system show conclusively that any slow motion in the system between $4 \mathrm{~K}$ and $20 \mathrm{~K}$ must have a characteristic time greater than $100 \mathrm{~ms}$. The second method allows one to probe the system on a longer time scale. It consists in measuring the damping of the stimulated echo following a sequence of three RF pulses, in which the waiting time $t_{w}$ 


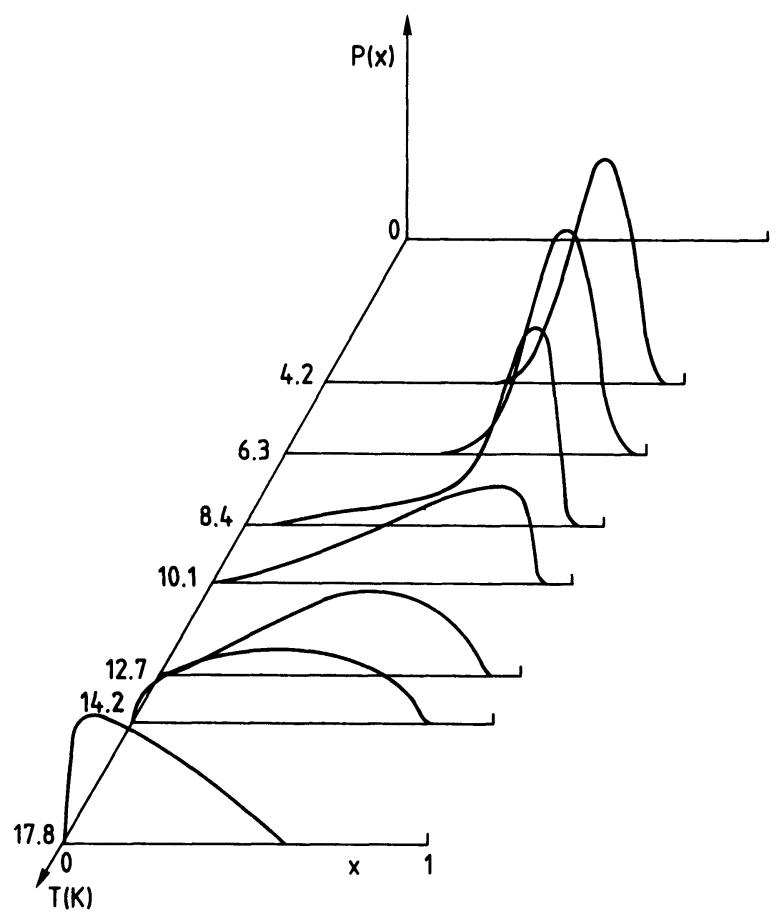

Fig. 2. - Evolution of the quadrupolarization distribution function $P(x)$ with the temperature as deduced from the analysis of the NMR line shapes.

before the third pulse is applied, is much greater than the time interval $\tau$ between the first and the second pulse (see Fig. 3). The two first pulses prepare the nuclear spins in a state of spin polarization and/or spin alignment which depends on the orientations of the local axes and of the values of the quadrupolarizations at that time. During the waiting time $t_{\mathrm{w}}$, the nuclear spins come back toward thermal equilibrium at a very slow rate since the dipolar flip-flop transitions between nuclei of different molecules are almost completely forbidden. A time $\tau$ after the third pulse which interrogates the nuclear spin states, a stimulated echo is focussed only if the molecular orientational states have not changed too much during the waiting time $t_{\mathrm{w}}$. In presence of a residual slow motion, the echo amplitude is affected by the following motional damping factor :

$$
A\left(t_{\mathrm{w}}\right)=\left\langle\frac{\cos (D+\chi) \Delta_{i}+\cos (D-\chi) \Delta_{i}}{2}\right\rangle
$$

with :

$$
\Delta_{i}=\left(\left\{x_{i} P_{2}\left(\alpha_{i}\right)\right\}_{t=0}-\left\{x_{i} P_{2}\left(\alpha_{i}\right)\right\}_{t=t_{w}}\right) \tau .
$$

For a slow motion of characteristic time $t_{\mathrm{m}}$, both terms appearing in (5) are respectively appreciably damped as soon as $t_{\mathrm{m}}$ is shorter than a limit time $t_{\mathrm{L} \pm}$ given by :

$$
t_{\mathrm{L} \pm}=(D \pm \chi)^{2}\left\langle x^{2}\right\rangle \tau^{2} t_{\mathrm{w}} .
$$

We have carried out a complete calculation of the damping factor $A\left(t_{\mathrm{w}}\right)$ in the case of a diffusive motion of the local axes $\hat{\zeta}_{i}$ which fully confirms this criterion. From the observed decay of the stimulated echoes one can infer a lower limit of $100 \mathrm{~s}$ for the characteristic time of any slow motion at all temperatures between $4 \mathrm{~K}$ and $20 \mathrm{~K}$. The observed damping is probably mainly due to the 


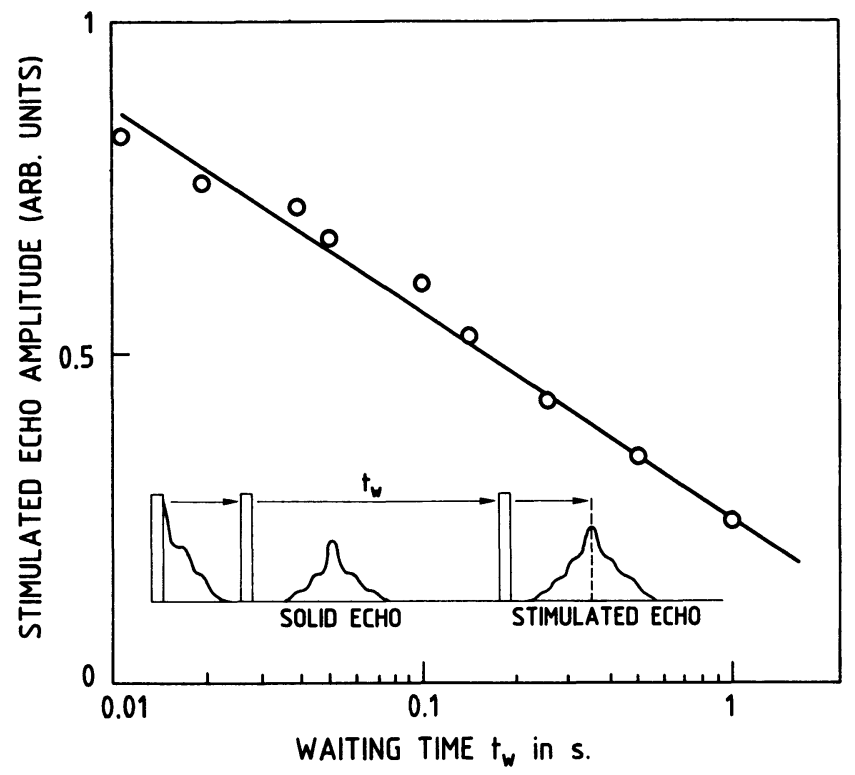

Fig. 3. - The observed decay of the stimulated echo following a three pulse RF sequence as a function of the waiting time $t_{\mathrm{w}}$ for a preparation time $\tau=2.75 \mathrm{~ms}$ at $T=5 \mathrm{~K}$. The weakness of the damping allows one to place a lower limit of $100 \mathrm{~s}$ on the characteristic time of any residual slow motion.

residual spectral diffusion during the waiting time, since its time dependence (a logarithmic law on a wide time scale) is similar to what has been observed for the long range ordered phase of pure f.c.c. nitrogen (Pa3 structure), for which any residual rotational motion is excluded. Our basic hypothesis of well defined orientational states is therefore fully confirmed by these stimulated echoes experiments.

3. An orientational glass regime. - The principal result of this NMR study for solid $\left(\mathrm{N}_{2}-\mathrm{Ar}\right)$ mixtures $\left(X_{\mathrm{N}_{2}}=0.675\right)$ is the observation of a continuous growth of the quadrupolarizations of the molecules as the temperature is reduced without any detectable thermodynamic transition $\left(^{1}\right)$. We call this low temperature regime an orientational glass regime since the degrees of freedom which are frozen are those describing the orientation of a classical rod. To what extent one faces a collective phenomenon is clearly the most important question to be answered. At high temperatures, the small quadrupolarizations one observes result from pair correlations induced by the quadrupolar interactions between the $\mathbf{N}_{2}$ molecules randomly distributed on the lattice. One would then expect [7] that on lowering the temperature, these correlations or similar ones involving only a few quadrupoles become stronger and that the system approaches its low temperature state continuously without any cooperative phenomenon taking place. This point of view seems in fact to be in contradiction to the results of recent Monte Carlo simulations of an assembly of interacting quadrupoles.

Previous work $[19,20]$ had already shown the presence of anomalously long evolution times in such a system. The MC experiments we have carried out [21] (random occupation $(X=0.67)$ of 96 and 216 sites on an h.c.p. lattice; up to $3 \times 10^{5} \mathrm{MCS}$ (Monte Carlo Steps) per quadrupole at a given temperature) have indicated that : (i) on lowering the temperature, there is at first

(1) Recent careful measurements of the specific heats of $\mathrm{N}_{2}$-Ar mixtures [23] (for $X_{\mathrm{N}_{2}}<0.75$ ) also show a smooth temperature dependence without any hint of transitional behaviour. 
a continuous growth of the local quadrupolarizations without appreciable modifications of the local axes $\hat{\zeta}_{i}$ until a cross-over towards a collective regime appears; (ii) this collective regime is characterized by correlated motions of the quadrupoles corresponding to jumps of the entire system between a few pockets in the configuration space, each pocket being associated with a ground state; (iii) on further lowering of the temperature, the system becomes trapped in a pocket. These results, although concerning a small system, show nevertheless that the onset of large quadrupolarizations in $\mathrm{N}_{2}$-Ar mixtures is associated with collective rotational motions of at least $10^{2}$ molecules.

Two types of collective behaviour have been identified in frustrated disordered systems, the so-called spin-glass and glass transitions. The evidence of a true transition in spin-glasses [22] (which is now supported by a large consensus [1]) arose from a careful examination of the magnetization and particularly of its field dependence. This type of experiment for quadrupole systems would correspond to measuring the response to an electrical field gradient and cannot be realized in a direct manner. In any case, the presence of quadrupolarizations at high temperatures would forbid measuring the analog of the zero field susceptibility. If one looks at dynamical properties, quadrupolar systems (at least ortho-para $\mathrm{H}_{2}$ mixtures [10]) and spin-glasses display a very substantial slowing-down in a narrow temperature range; but evidence of a true transition cannot be inferred from it.

For conventional glasses, one does not observe a sharp transition in static properties but only a very rapid slowing-down. The glass state is characterized by strong time dependent effects (such as enthalpy and volume relaxation), and by the now well known two level systems. Such features have been alluded to in studies of quadrupolar systems $[6,10]$ but never studied in depth. Whether the orientational glass is a true equilibrium state (as now admitted for the low temperature state of spin-glasses), or a non-equilibrium state (as for conventional glasses) remains unknown, but experiments designed to answer this question are planned in different laboratories.

4. Conclusion. - Despite their very particular natures, the quadrupolar systems $\left(\mathrm{N}_{2}-\mathrm{Ar}\right.$, ortho-para $\mathrm{H}_{2}$ mixtures) form glass-like states (orientational and quadrupolar glasses) with properties very similar to those observed for ordinary glasses and spin-glasses. Since the only conceptual link between these very different systems is that they incorporate both frustration and disorder, the similarity in their behaviour supports the argument that the combination of these basic ingredients is essential for glass formation.

Note added in proof. - The results of recent neutron scattering experiments on $\mathrm{N}_{2}-\mathrm{Ar}$ alloys by $\mathrm{W}$. Press and colleagues are in qualitative agreement with the conclusions given here and we thank W. Press for communicating his results prior to publication (PRESs, W., JANIK and GrIMM, to be published in Z. Phys. (1982)).

\section{References}

[1] For a general review of this subject see :

ToulousE, G., « Frustrations et désordres : problèmes nouveaux en mécanique statistique, histoire des verres de spin $"$ in Congrès de la Société Française de Physique (Editions de Physique) 1981, p. 3.

[2] SCOTT, T. A., Phys. Rep. 27 (1976) 89.

[3] Silvera, I. F., Rev. Mod. Phys. 52 (1980) 393 and references therein.

[4] Sullivan, N. S., Devoret, M., Cowan, B. P. and Urbina, C., Phys. Rev. B 17 (1978) 5016.

[5] Estève, D., Devoret, M. and Sullivan, N. S., J. Phys. C (Solid State), to be published.

[6] Candela, D., Buchman, S., Vetterling, W. T. and Pound, R. V., Physica B + C 107 (1981) 187.

[7] Washburn, S., Calkins, M., Meyer, H. and Harris, A. B., to be published. 
[8] Ishimoto, S., Nagamine, K., Kimura, Y. and Kumagi, H., J. Phys. Soc. Japan 40 (1976) 312.

[9] Cochran, W. T., Gaines, J. R., Mc Call, R. P., Sokol, P. E. and Patton, B. R., Phys. Rev. Lett. 45 (1980) 1576.

[10] Sullivan, N. S. and Estève, D., Physica $B+C 107$ (1981) 189.

[11] HaAse, D. G. and Saleh, A. M., Physica $B+C 107$ (1981) 191.

[12] Sullivan, N. S., Deyoret, M. and Vaissière, J. M., J. Physique-Lett. 40 (1979) L-559.

[13] Gates, J. V., Granfors, P. R., Fraas, B. A. and Simmons, R. O., Phys. Rev. B 19 (1979) 3667.

[14] Barret, C. S. and Meyer, L., J. Chem. Phys. 42 (1965) 107.

[15] Pace, E. L., Smith, J. H. and Jepson, B. E., J. Chem. Phys. 50 (1969) 312.

[16] Ishol, L. M. and Scott, T. A., J. Magn. Res. 27 (1977) 23.

[17] Estève, D. and Sullivan, N. S., J. Phys. C (Solid State), to be published.

[18] Sullivan, N. S., Estève, D. and Devoret, M., J. Phys. C (Solid State), to be published.

[19] Mandell, M., J. Chem. Phys. 60 (1974) 4880.

[20] Klenin, M. A. and Pate, S. F., Physica B + C 107 (1981) 185.

[21] Devoret, M. and Estève, D., to be published.

[22] Berton, A., Chaussy, J., Odin, J., Rammal, R. and Tournier, R., J. Physique-Lett. 43 (1982) L-153.

[23] HAASE, D. G., Private communication. 